\title{
High Degree Vertices and Eigenvalues in the Preferential Attachment Graph
}

\author{
Abraham Flaxman, Alan Frieze, and Trevor Fenner
}

Abstract. The preferential attachment graph is a random graph formed by adding a new vertex at each time-step, with a single edge which points to a vertex selected at random with probability proportional to its degree. Every $m$ steps the most recently added $m$ vertices are contracted into a single vertex, so at time $t$ there are roughly $t / m$ vertices and exactly $t$ edges. This process yields a graph which has been proposed as a simple model of the World Wide Web [Barabási and Albert 99]. For any constant $k$, let $\Delta_{1} \geq \Delta_{2} \geq \cdots \geq \Delta_{k}$ be the degrees of the $k$ highest degree vertices. We show that at time $t$, for any function $f$ with $f(t) \rightarrow \infty$ as $t \rightarrow \infty, \frac{t^{1 / 2}}{f(t)} \leq \Delta_{1} \leq t^{1 / 2} f(t)$, and for $i=2, \ldots, k, \frac{t^{1 / 2}}{f(t)} \leq \Delta_{i} \leq \Delta_{i-1}-\frac{t^{1 / 2}}{f(t)}$, with high probability (whp). We use this to show that at time $t$ the largest $k$ eigenvalues of the adjacency matrix of this graph have $\lambda_{k}=(1 \pm o(1)) \Delta_{k}^{1 / 2}$ whp.

\section{Introduction}

Recently, there has been much interest in understanding the properties of realworld large-scale networks such as the structure of the Internet and the World Wide Web. For a general introduction to this topic, see Bollobás and Riordan [Bollobás and Riordan 02], Hayes [Hayes 00], or Watts [Watts 99]. One approach is to model these networks by random graphs. Experimental studies by Albert, Barabási, and Jeong [Albert et al. 99], Broder et al. [Broder et al. 00], and Faloutsos, Faloutsos, and Faloutsos [Faloutsos et al. 99] have demonstrated that

(C) A K Peters, Ltd.

I542-795I/05 \$0.50 per page 
in the World Wide Web/Internet the proportion of vertices of a given degree follows an approximate inverse power law, i.e., the proportion of vertices of degree $k$ is approximately $C k^{-\alpha}$ for some constants $C, \alpha$. The classical models of random graphs introduced by Erdős and Renyi [Erdös and Rényi 59] do not have power law degree sequences, so they are not suitable for modeling these networks. This has driven the development of various alternative models for random graphs.

One approach to remedy this situation is to study graphs with a prescribed degree sequence (or prescribed expected degree sequence). This is proposed as a model for the web graph by Aiello, Chung, and Lu in [Aiello et al. 00]. Mihail and Papadimitriou also use this model [Mihail and Papadimitriou 02] in their study of large eigenvalues, as do Chung, Lu, and $\mathrm{Vu}$ in [Chung et al. 03a, Chung et al. 03b].

An alternative approach, which we will follow in this paper, is to sample graphs via some generative procedure which yields a power law distribution. There is a long history of such models, outlined in the survey by Mitzenmacher [Mitzenmacher 04]. We will use the preferential attachment model to generate our random graph. The preferential attachment random graph has been the subject of recently revived interest. It dates back to Yule [Yule 25] and Simon [Simon 55]. It was proposed as a model for the web by Barabási and Albert [Barabási and Albert 99], and their description was elaborated by Bollobás and Riordan in [Bollobás and Riordan]. It was used by Bollobás, Riordan, Spencer, and Tusnády [Bollobás et al. 01], who proved that the degree sequence does follow a power law distribution. Bollobás and Riordan obtained several additional results regarding the diameter and connectivity of such graphs [Bollobás and Riordan]. We use the generative model of [Bollobás and Riordan] (see also [Bollobás et al. 01]) and build a graph sequentially as follows:

- At each time-step $t$, we add a vertex $v_{t}$, and we add an edge from $v_{t}$ to some other vertex $u$, where $u$ is chosen at random according to the distribution:

$$
\operatorname{Pr}\left[u=v_{i}\right]= \begin{cases}\frac{d_{t}\left(v_{i}\right)}{2 t-1}, & \text { if } v_{i} \neq v_{t} ; \\ \frac{1}{2 t-1}, & \text { if } v_{i}=v_{t}\end{cases}
$$

where $d_{t}(v)$ denotes the degree of vertex $v$ at time $t$. This means that each vertex receives an additional edge with probability proportional to its current degree. The probability of choosing $v_{t}$ (and forming a loop) is consistent with this, since we've already committed "half" an edge to $v_{t}$ and are deciding where to put the other half.

- For some constant $m$, every $m$ steps we contract the most recently added $m$ vertices to form a supervertex. 
Let $G_{t}^{m}$ denote the random graph at time-step $t$ with contractions of size $m$. Note that contracting each set of vertices $\{i m+1, i m+2, \ldots,(i+1) m\}$ of $G_{t}^{1}$ yields a graph identically distributed with $G_{t}^{m}$.

It is worth mentioning that there are several alternative simple models for the World Wide Web and for general power law graphs. A generalization of the preferential attachment model is described by Drinea, Enachescu, and Mitzenmacher in [Drinea et al. 01], and degree sequence results analogous to [Bollobás et al. 01] are proved for this model by Buckley and Osthus in [Buckley and Osthus]. A completely different generative model, based on the idea that new web pages are often consciously or unconsciously copies of existing pages, is developed by Kleinberg et al. and Kumar et al. in [Kleinberg et al. 99], [Kumar et al. 99], [Kumar et al. 00]. Cooper and Frieze analyze a model combining these approaches in [Cooper and Frieze 01].

Several previous results have studied the structure of low-degree vertices in the preferential attachment graph. For example, the results in [Bollobás et al. 01] concern degrees up to $t^{1 / 15}$. The maximum degree vertex of the preferential attachment graph is the subject of Theorem 17 of [Bollobás and Riordan 02], where an elegant static description of the preferential attachment graph is used to show that $\Delta_{1} / \sqrt{t}$ converges in distribution to a certain nonnegative distribution. The technique used there extends to give the asymptotic distribution of $\Delta_{i} / \sqrt{t}$ for any constant $i$. Our first theorem also deals with the highest degree vertices:

Theorem I.I. Let $m$ and $k$ be fixed positive integers, and let $f(t)$ be a function with $f(t) \rightarrow \infty$ as $t \rightarrow \infty$. Let $\Delta_{1} \geq \Delta_{2} \geq \cdots \geq \Delta_{k}$ denote the degrees of the $k$ highest degree vertices of $G_{t}^{m}$. Then

$$
\frac{t^{1 / 2}}{f(t)} \leq \Delta_{1} \leq t^{1 / 2} f(t)
$$

and for $i=2, \ldots, k$,

$$
\frac{t^{1 / 2}}{f(t)} \leq \Delta_{i} \leq \Delta_{i-1}-\frac{t^{1 / 2}}{f(t)}
$$

whp. ${ }^{1}$

Unfortunately, the slowly growing function $f(t)$ in the result above cannot be removed. Indeed, Theorem 17 of [Bollobás and Riordan 02] and its extension to the $k$ largest degrees shows that for any constants $a<b$ we have

$$
\lim _{t \rightarrow \infty} \operatorname{Pr}\left[\Delta_{1} \in\left(a t^{1 / 2}, b t^{1 / 2}\right)\right]>0 \text { and } \lim _{t \rightarrow \infty} \operatorname{Pr}\left[\Delta_{i}-\Delta_{i-1} \in\left(a t^{1 / 2}, b t^{1 / 2}\right)\right]>0 .
$$

\footnotetext{
${ }^{1}$ In this paper, an event $\mathcal{E}$ is said to hold with high probability (whp) if $\operatorname{Pr}[\mathcal{E}] \rightarrow 1$ as $t \rightarrow \infty$.
} 
The next theorem relates maximum eigenvalues and maximum degrees. It mirrors results of Mihail and Papadimitriou [Mihail and Papadimitriou 02] and Chung, Liu and $\mathrm{Vu}$ [Chung et al. 03a, Chung et al. 03b] for fixed degree expectation models and at a high level, the proof follows the same lines as these two papers. Experimentally, a power law distribution for eigenvalues was observed in "real-world" graphs in [Faloutsos et al. 99].

Theorem 1.2. Let $m$ and $k$ be fixed positive integers, and let $f(t)$ be a function with $f(t) \rightarrow \infty$ as $t \rightarrow \infty$. Let $\lambda_{1} \geq \lambda_{2} \geq \cdots \geq \lambda_{k}$ be the $k$ largest eigenvalues of the adjacency matrix of $G_{t}^{m}$. Then for $i=1, \ldots, k$ we have $\lambda_{i}=(1 \pm o(1)) \Delta_{i}^{1 / 2}$ whp.

Our proofs of these theorems require two lemmas.

Lemma 1.3. Let $d_{t}^{m}(s)$ denote the degree of vertex $s$ in $G_{t}^{m}$, and let $a^{(k)}=a(a+$ $1)(a+2) \cdots(a+k-1)$ denote the rising factorial function. Then for any positive integer $k$,

$$
\mathrm{E}\left[\left(d_{t}^{m}(s)\right)^{(k)}\right] \leq(2 m)^{(k)} 2^{k / 2}\left(\frac{t}{s}\right)^{k / 2}
$$

To simplify the exposition, we speak of a supernode, which is simply a collection of vertices viewed as one vertex. So the degree of a supernode is the sum of the degrees of the vertices in the supernode, and an edge is incident to a supernode if it is incident to some vertex in the supernode.

Lemma I.4. Let $\mathbf{S}=\left(S_{1}, S_{2}, \ldots, S_{\ell}\right)$ be a collection of disjoint supernodes, and let $p_{\mathbf{S}}\left(\mathbf{r} ; \mathbf{d}, t_{0}, t\right)$ denote the probability that each supernode $S_{i}$ has degree $r_{i}+d_{i}$ at time $t$ conditioned on $d_{t_{0}}\left(S_{i}\right)=d_{i}$. Let $d=\sum_{i=1}^{\ell} d_{i}$ and $r=\sum_{i=1}^{\ell} r_{i}$. If $d=o\left(t^{1 / 2}\right)$ and $r=o\left(t^{2 / 3}\right)$, then

$$
p_{\mathbf{S}}\left(\mathbf{r} ; \mathbf{d}, t_{0}, t\right) \leq\left(\prod_{i=1}^{\ell}\left(\begin{array}{c}
r_{i}+d_{i}-1 \\
d_{i}-1
\end{array}\right)\right)\left(\frac{t_{0}+1}{t}\right)^{d / 2} \exp \left\{2+t_{0}-\frac{d}{2}+\frac{2 r}{t^{1 / 2}}\right\}
$$

In the next section, we prove Lemma 1.3, Lemma 1.4, and Theorems 1.1 and 1.2 . 


\section{Proofs}

\section{I. Proof of Lemma I.3}

An earlier version of the paper bounded $\mathrm{E}\left[\left(d_{t}^{m}(s)\right)^{k}\right]$. This was a quite involved calculation. One of the referees suggested that we bound $\mathrm{E}\left[\left(d_{t}^{m}(s)\right)^{(k)}\right]$ because this would be simpler using an idea from [Bollobás and Riordan 02]. This is indeed the case, as the reader can see next.

Let $Z_{t}=d_{t}^{m}(s)$ denote the degree of vertex $s$ at time $t$ (when the graph contains $t$ edges), and let $Y_{t}$ be an indicator variable for the event that the edge added at time $t$ is incident to $s$.

Then we have

$$
\begin{aligned}
\mathrm{E}\left[Z_{t}^{(k)}\right] & =\mathrm{E}\left[\mathrm{E}\left[\left(Z_{t-1}+Y_{t}\right)^{(k)}\right] \mid Z_{t-1}\right] \\
& =\mathrm{E}\left[Z_{t-1}^{(k)}\left(1-\frac{Z_{t-1}}{2 t-1}\right)+\left(Z_{t-1}+1\right)^{(k)}\left(\frac{Z_{t-1}}{2 t-1}\right)\right] \\
& =\left(1+\frac{k}{2 t-1}\right) \mathrm{E}\left[Z_{t-1}^{(k)}\right] .
\end{aligned}
$$

Since $Z_{s}^{(k)} \leq(2 m)^{(k)}$, we have

$$
\mathrm{E}\left[Z_{t}^{(k)}\right] \leq(2 m)^{(k)} \prod_{t^{\prime}=s+1}^{t}\left(1+\frac{k}{2 t^{\prime}-1}\right) \leq(2 m)^{(k)} \exp \left\{\frac{k}{2} \sum_{t^{\prime}=s+1}^{t} \frac{1}{t^{\prime}-\frac{1}{2}}\right\} .
$$

We upper bound the sum with an integral,

$$
\sum_{t^{\prime}=s+1}^{t} \frac{1}{t^{\prime}-\frac{1}{2}} \leq \int_{x=s}^{t} \frac{1}{x-\frac{1}{2}} d x=\log \frac{t-\frac{1}{2}}{s-\frac{1}{2}}
$$

and the bound on the expectation becomes

$$
\mathrm{E}\left[Z_{t}^{(k)}\right] \leq(2 m)^{(k)}\left(\frac{t-\frac{1}{2}}{s-\frac{1}{2}}\right)^{k / 2}=(2 m)^{(k)}\left(\frac{t}{s}\right)^{k / 2}\left(\frac{2-1 / t}{2-1 / s}\right)^{k / 2} .
$$

Since $\frac{2-1 / t}{2-1 / s} \leq 2$, we may conclude that

$$
\mathrm{E}\left[Z_{t}^{(k)}\right] \leq(2 m)^{(k)} 2^{k / 2}\left(\frac{t}{s}\right)^{k / 2}
$$




\subsection{Proof of Lemma I.4}

We calculate the probability as the union of disjoint events by fixing the times when the degrees of the $S_{i}$ change. Let $\tau^{(i)}=\left(\tau_{1}^{(i)}, \ldots, \tau_{r_{i}}^{(i)}\right)$, where $\tau_{j}^{(i)}$ is the time when we add an edge incident to $S_{i}$ and increase the degree of $S_{i}$ from $d_{i}+j-1$ to $d_{i}+j$. We will see that in the calculation it doesn't matter much which $S_{i}$ increases in degree, so we let $d=\sum_{i=1}^{\ell} d_{i}$ and $r=\sum_{i=1}^{\ell} r_{i}$ and define $\tau=\left(\tau_{0}, \tau_{1}, \ldots, \tau_{r+1}\right)$ to be the ordered union of the $\tau^{(i)}$, with $\tau_{0}=t_{0}$ and $\tau_{r+1}=t$.

Let $p\left(\tau ; \mathbf{d}, t_{0}, t\right)$ denote the probability that (super)nodes $S_{i}$ increase in degree at exactly the times specified by $\tau$ between time $t_{0}$ and $t$ given $d_{t_{0}}\left(s_{i}\right)=d_{i}$. Then

$$
\begin{aligned}
p\left(\tau ; \mathbf{d}, t_{0}, t\right)= & \left(\prod_{i=1}^{\ell} \prod_{k=1}^{r_{i}} \frac{d_{i}+k-1}{2 \tau_{k}^{(i)}-1}\right)\left(\prod_{k=0}^{r} \prod_{j=\tau_{k}+1}^{\tau_{k+1}-1}\left(1-\frac{d+k}{2 j-1}\right)\right) \\
= & \left(\prod_{i=1}^{\ell} \frac{\left(r_{i}+d_{i}-1\right) !}{\left(d_{i}-1\right) !}\right)\left(\prod_{k=1}^{r} \frac{1}{2 \tau_{k}-1}\right) \\
& \times \exp \left\{\sum_{k=0}^{r} \sum_{j=\tau_{k}+1}^{\tau_{k+1}-1} \log \left(1-\frac{d+k}{2 j-1}\right)\right\} .
\end{aligned}
$$

We bound the inner sum by an integral

$$
\begin{aligned}
\sum_{j=\tau_{k}+1}^{\tau_{k+1}-1} \log \left(1-\frac{d+k}{2 j-1}\right) & \leq \sum_{j=\tau_{k}+1}^{\tau_{k+1}-1} \log \left(1-\frac{d+k}{2 j}\right) \\
& \leq \int_{\tau_{k}+1}^{\tau_{k+1}} \log \left(1-\frac{d+k}{2 x}\right) d x
\end{aligned}
$$

Then, since

$$
\int \log \left(1-\frac{d+k}{2 x}\right) d x=-x \log (2 x)+\frac{2 x-(d+k)}{2} \log (2 x-(d+k)),
$$

we have

$$
\begin{aligned}
\int_{\tau_{k}+1}^{\tau_{k+1}} \log \left(1-\frac{d+k}{2 x}\right) d x & \\
=- & \tau_{k+1} \log \left(2 \tau_{k+1}\right)+\frac{2 \tau_{k+1}-(d+k)}{2} \log \left(2 \tau_{k+1}-(d+k)\right) \\
& \quad+\left(\tau_{k}+1\right) \log \left(2 \tau_{k}+2\right)-\frac{2 \tau_{k}+2-(d+k)}{2} \log \left(2 \tau_{k}+2-(d+k)\right) .
\end{aligned}
$$


By grouping like terms and noting that $\tau_{0}=t_{0}$ and $\tau_{r+1}=t$, we have

$$
\begin{aligned}
& \sum_{k=0}^{r} \int_{\tau_{k}+1}^{\tau_{k+1}} \log \left(1-\frac{d+k}{2 x}\right) d x \\
& =\left(t_{0}+1\right) \log \left(2 t_{0}+2\right)-\frac{2 t_{0}+2-d}{2} \log \left(2 t_{0}+2-d\right) \\
& \quad-t \log (2 t)+\frac{2 t-(d+r)}{2} \log (2 t-(d+r)) \\
& \quad+\sum_{k=1}^{r}\left(\left(\tau_{k}+1\right) \log \left(2 \tau_{k}+2\right)-\frac{2 \tau_{k}+2-(d+k)}{2} \log \left(2 \tau_{k}+2-(d+k)\right)\right. \\
& \left.\quad-\tau_{k} \log \left(2 \tau_{k}\right)+\frac{2 \tau_{k}-(d+k-1)}{2} \log \left(2 \tau_{k}-(d+k-1)\right)\right) \\
& =A+\sum_{k=1}^{r} B_{k},
\end{aligned}
$$

where $A$ is the term outside the summation and $B_{k}$ is the $k$ th term of the sum.

We concentrate first on the term $B_{k}$. Rearranging terms yields

$$
\begin{aligned}
B_{k}= & \tau_{k} \log \left(1+1 / \tau_{k}\right) \\
& +\log \left(2 \tau_{k}+2\right)+\frac{2 \tau_{k}+2-(d+k)}{2} \log \left(1-\frac{1}{2 \tau_{k}+2-(d+k)}\right) \\
& -\frac{1}{2} \log \left(2 \tau_{k}+1-(d+k)\right) .
\end{aligned}
$$

Since $1+x \leq e^{x}$, this is bounded as

$$
B_{k} \leq \frac{1}{2} \log \left(2 \tau_{k}+2\right)-\frac{1}{2} \log \left(1-\frac{d+k+1}{2 \tau_{k}+2}\right)+\frac{1}{2}
$$

Now we turn our attention to $A$. Rearranging terms, we have

$$
\begin{gathered}
A=-\left(t_{0}+1\right) \log \left(1-\frac{d}{2 t_{0}+2}\right)+\frac{d}{2} \log \left(2 t_{0}+2-d\right)+t \log \left(1-\frac{d+r}{2 t}\right) \\
-\frac{d+r}{2} \log (2 t-(d+r)) .
\end{gathered}
$$


So

$$
\begin{aligned}
e^{A}= & \left(1-\frac{d}{2 t_{0}+2}\right)^{-\left(t_{0}+1\right)}\left(2 t_{0}+2-d\right)^{d / 2}\left(1-\frac{d+r}{2 t}\right)^{t} \\
& \times(2 t-(d+r))^{-(d+r) / 2} \\
= & \left(1-\frac{d}{2\left(t_{0}+1\right)}\right)^{-\left(1-\frac{d}{2\left(t_{0}+1\right)}\right)\left(t_{0}+1\right)} \\
& \times\left(1-\frac{d+r}{2 t}\right)^{t-(d+r) / 2}\left(\frac{t_{0}+1}{t}\right)^{d / 2}(2 t)^{-r / 2}
\end{aligned}
$$

Since $1-x \leq e^{-x-x^{2} / 2}$ for $0<x<1$, we have

$$
\left(1-\frac{d+r}{2 t}\right)^{t-(d+r) / 2} \leq \exp \left\{-\frac{d+r}{2}+\frac{(d+r)^{2}}{8 t}+\frac{(d+r)^{3}}{16 t^{2}}\right\}
$$

So

$$
\begin{aligned}
e^{A+\sum B_{k} \leq} \leq & \left.1-\frac{d}{2\left(t_{0}+1\right)}\right)^{-\left(1-\frac{d}{2\left(t_{0}+1\right)}\right)\left(t_{0}+1\right)} \\
& \times \exp \left\{-\frac{d+r}{2}+\frac{(d+r)^{2}}{8 t}+\frac{(d+r)^{3}}{16 t^{2}}\right\} \\
& \times\left(\frac{t_{0}+1}{t}\right)^{d / 2}(2 t)^{-r / 2} \\
& \times\left(\prod_{k=1}^{r}\left(\left(1-\frac{d+k+1}{2 \tau_{k}+2}\right)^{-1 / 2}\left(2 \tau_{k}+2\right)^{1 / 2}\right)\right) e^{r / 2} \\
= & \operatorname{err}\left(r, d, t_{0}, t\right)\left(\frac{t_{0}+1}{t}\right)^{d / 2}(2 t)^{-r / 2} \\
& \times\left(\prod_{k=1}^{r}\left(\left(1-\frac{d+k+1}{2 \tau_{k}+2}\right)^{-1 / 2}\left(2 \tau_{k}+2\right)^{1 / 2}\right)\right),
\end{aligned}
$$

where

$$
\begin{aligned}
\operatorname{err}\left(r, d, t_{0}, t\right)= & \left(1-\frac{d}{2\left(t_{0}+1\right)}\right)^{-\left(1-\frac{d}{2\left(t_{0}+1\right)}\right)\left(t_{0}+1\right)} \\
& \exp \left\{-\frac{d}{2}+\frac{(d+r)^{2}}{8 t}+\frac{(d+r)^{3}}{16 t^{2}}\right\} .
\end{aligned}
$$


Inserting the bounds for $A+\sum B_{k}$ into the bound on $p\left(\tau ; \mathbf{d}, t_{0}, t\right)$, we have

$$
\begin{aligned}
p\left(\tau ; \mathbf{d}, t_{0}, t\right) \leq & \left(\prod_{i=1}^{\ell} \frac{\left(r_{i}+d_{i}-1\right) !}{\left(d_{i}-1\right) !}\right) \operatorname{err}\left(r, d, t_{0}, t\right)\left(\frac{t_{0}+1}{t}\right)^{d / 2}(2 t)^{-r / 2} \\
& \times\left(\prod_{k=1}^{r}\left(1-\frac{d+k+1}{2 \tau_{k}+2}\right)^{-1 / 2}\left(2 \tau_{k}+2\right)^{1 / 2}\left(2 \tau_{k}-1\right)^{-1}\right) .
\end{aligned}
$$

Now observe that

$$
\begin{aligned}
\left(1-\frac{d+k+1}{2 \tau_{k}+2}\right)^{-1 / 2}\left(2 \tau_{k}+2\right)^{1 / 2}\left(2 \tau_{k}-1\right)^{-1} & = \\
& \left(2 \tau_{k}+1-(d+k)\right)^{-1 / 2}\left(1+\frac{3}{2 \tau_{k}-1}\right) .
\end{aligned}
$$

In order to bound the probability of interest, we sum $p\left(\tau ; \mathbf{d}, t_{0}, t\right)$ over all ordered choices of $\tau$ :

$$
\begin{aligned}
p_{\mathbf{S}}\left(\mathbf{r} ; \mathbf{d}, t_{0}, t\right)= & \sum_{\tau^{(1)}, \ldots, \tau^{(\ell)}} p\left(\tau ; \mathbf{d}, t_{0}, t\right) \\
\leq & \left(\begin{array}{c}
r \\
r_{1}, \ldots, r_{\ell}
\end{array}\right) \sum_{t_{0}+1 \leq \tau_{1}<\cdots<\tau_{r} \leq t}\left(\prod_{i=1}^{\ell} \frac{\left(r_{i}+d_{i}-1\right) !}{\left(d_{i}-1\right) !}\right) \operatorname{err}\left(r, d, t_{0}, t\right) \\
& \times\left(\frac{t_{0}+1}{t}\right)^{d / 2}(2 t)^{-r / 2} \\
& \times\left(\prod_{k=1}^{r}\left(2 \tau_{k}+1-(d+k)\right)^{-1 / 2}\left(1+\frac{3}{2 \tau_{k}-1}\right)\right) \\
= & r !\left(\prod_{i=1}^{\ell}\left(\begin{array}{l}
r_{i}+d_{i}-1 \\
d_{i}-1
\end{array}\right)\right) \operatorname{err}\left(r, d, t_{0}, t\right)\left(\frac{t_{0}+1}{t}\right)^{d / 2}(2 t)^{-r / 2} \\
& \times \sum_{t_{0}+1 \leq \tau_{1}<\tau_{2} \cdots<\tau_{r} \leq t}\left(\prod_{k=1}^{r}\left(2 \tau_{k}+1-(d+k)\right)^{-1 / 2}\left(1+\frac{3}{2 \tau_{k}-1}\right)\right) .
\end{aligned}
$$

Now we make a change of variables, introducing $\tau_{k}^{\prime}=\tau_{k}-\lceil(d+k) / 2\rceil$. For some $\tau_{k}, \tau_{k+1}$, this can result in $\tau_{k}^{\prime}=\tau_{k+1}^{\prime}$, so we relax the strict inequalities to lessthan-or-equals. Also, since $d$ and $k$ are both at least 1 , we have $2\lceil(d+k) / 2\rceil \geq 2$. 
So

$$
\begin{aligned}
& \sum_{t_{0}<\tau_{1}<\tau_{2}<\cdots<\tau_{r} \leq t}\left(\prod_{k=1}^{r}\left(2 \tau_{k}+1-(d+k)\right)^{-1 / 2}\left(1+\frac{3}{2 \tau_{k}-1}\right)\right) \\
& \leq \sum_{\left(t_{0}-\lceil d / 2\rceil+1\right) \leq \tau_{1}^{\prime} \leq \tau_{2}^{\prime} \leq \cdots \leq \tau_{r}^{\prime} \leq(t-\lceil(d+r) / 2\rceil)}\left(\prod_{k=1}^{r}\left(2 \tau_{k}^{\prime}+1\right)^{-1 / 2}\left(1+\frac{3}{2 \tau_{k}^{\prime}+1}\right)\right) .
\end{aligned}
$$

We simplify this sum by unordering the variables,

$$
\begin{gathered}
\sum_{\left(t_{0}-\lceil d / 2\rceil+1\right) \leq \tau_{1}^{\prime} \leq \tau_{2}^{\prime} \leq \cdots \leq \tau_{r}^{\prime} \leq(t-\lceil(d+r) / 2\rceil)}\left(\prod_{k=1}^{r}\left(\left(2 \tau_{k}^{\prime}+1\right)^{-1 / 2}+3\left(2 \tau_{k}+1\right)^{-3 / 2}\right)\right) \\
=\frac{1}{r !}\left(\sum_{\tau^{\prime}=t_{0}-\lceil d / 2\rceil+1}^{t-\lceil(d+r) / 2\rceil}\left(\left(2 \tau^{\prime}+1\right)^{-1 / 2}+3\left(2 \tau^{\prime}+1\right)^{-3 / 2}\right)\right)^{r},
\end{gathered}
$$

and then using an integral, which we start from $x=0$, since $t_{0}-\lceil d / 2\rceil+1 \geq 1$,

$$
\begin{aligned}
& \sum_{\tau^{\prime}=t_{0}-\lceil d / 2\rceil+1}^{t-\lceil(d+r) / 2\rceil}\left(2 \tau^{\prime}+1\right)^{-1 / 2}+3\left(2 \tau^{\prime}+1\right)^{-3 / 2} \\
& \quad \leq \int_{x=0}^{t-(d+r) / 2}\left((2 x+1)^{-1 / 2}+3(2 x+1)^{-3 / 2}\right) d x \\
& \left.\leq(2 t-(d+r)+1)^{1 / 2}-1-3(2 t-(d+r)+1)^{-1 / 2}+3\right) \\
& \leq(2 t-(d+r)+1)^{1 / 2}+2 \\
& \quad=(2 t)^{1 / 2}\left(1-\frac{d+r-1}{2 t}\right)^{1 / 2}\left(1+\frac{2}{(2 t-(d+r)+1)^{1 / 2}}\right) .
\end{aligned}
$$

Again using $1+x \leq e^{x}$, we have

$$
\left(1-\frac{d+r-1}{2 t}\right)^{r / 2} \leq \exp \left\{-\frac{r(d+r-1)}{4 t}\right\}
$$

and

$$
\left(1+\frac{2}{(2 t-(d+r)+1)^{1 / 2}}\right)^{r} \leq \exp \left\{\frac{2 r}{(2 t-(d+r)+1)^{1 / 2}}\right\} .
$$

So

$$
\begin{aligned}
p_{\mathbf{S}}\left(\mathbf{r} ; \mathbf{d}, t_{0}, t\right) \leq & \left(\prod_{i=1}^{\ell}\left(\begin{array}{c}
r_{i}+d_{i}-1 \\
d_{i}-1
\end{array}\right)\right) \operatorname{err}\left(r, d, t_{0}, t\right)\left(\frac{t_{0}+1}{t}\right)^{d / 2} \\
& \exp \left\{-\frac{r(d+r-1)}{4 t}+\frac{2 r}{(2 t-(d+r)+1)^{1 / 2}}\right\} .
\end{aligned}
$$


For $d=o\left(t^{1 / 2}\right)$ and $r=o\left(t^{2 / 3}\right)$, we have

$$
\begin{aligned}
& \operatorname{err}\left(r, d, t_{0}, t\right) \exp \left\{-\frac{r(d+r-1)}{4 t}+\frac{2 r}{(2 t-(d+r)+1)^{1 / 2}}\right\} \\
& \quad \leq\left(1-\frac{d}{2\left(t_{0}+1\right)}\right)^{-\left(1-\frac{d}{2\left(t_{0}+1\right)}\right)\left(t_{0}+1\right)} \exp \left\{1-\frac{d}{2}-\frac{r^{2}}{8 t}+\frac{2 r}{t^{1 / 2}}\right\} .
\end{aligned}
$$

Since $x^{-x} \leq e$, this completes the proof of the lemma.

\subsection{Proof of Theorem I.I}

We partition the vertices into those added before time $t_{0}$, before $t_{1}$, and after $t_{1}$ and argue about the maximum degree of vertices in each set. Here,

$$
t_{0}=\log \log \log f(t) \text { and } t_{1}=\log \log f(t) .
$$

We break the proof of Theorem 1.1 into five claims.

Claim 2.I. In $G_{t}^{m}$, the degree of the supernode of vertices added before time $t_{0}$ is at least $t_{0}^{1 / 3} t^{1 / 2}$ whp.

Proof. Let $\mathcal{A}_{1}$ denote the event that the supernode consisting of the first $t_{0}$ vertices has degree less than $t_{0}^{1 / 3} t^{1 / 2}$. We bound the probability of this event using Lemma 1.4 with $\ell=1$. Since at time $t_{0}$ the supernode of all vertices added by this time has all of the edges, we take $\mathbf{d}=d_{1}=2 t_{0}$. Then

$$
\begin{aligned}
\operatorname{Pr}\left[\mathcal{A}_{1}\right] & \leq \sum_{r_{1}=0}^{t_{0}^{1 / 3} t^{1 / 2}-2 t_{0}}\left(\begin{array}{c}
r_{1}+2 t_{0}-1 \\
2 t_{0}-1
\end{array}\right)\left(\frac{t_{0}+1}{t}\right)^{d / 2} e^{2+t_{0}-d / 2+2 r / t^{1 / 2}} \\
& \leq\left(t_{0}^{1 / 3} t^{1 / 2}\right) \frac{\left(t_{0}^{1 / 3} t^{1 / 2}\right)^{2 t_{0}-1}}{\left(2 t_{0}-1\right) !}\left(\frac{t_{0}+1}{t}\right)^{t_{0}} e^{2+t_{0}+2 t_{0}^{1 / 3}} \\
& \leq t_{0}^{2 t_{0} / 3} \frac{e^{2 t_{0}-1}}{\left(2 t_{0}-1\right)^{2 t_{0}-1}}\left(t_{0}+1\right)^{t_{0}} e^{2+t_{0}+2 t_{0}^{1 / 3}} \\
& \leq \frac{e^{3 t_{0}+2 t_{0}^{1 / 3}+2}}{\left(2 t_{0}-1\right)^{t_{0} / 3-1}} \\
& =o(1) .
\end{aligned}
$$

Claim 2.2. In $G_{t}^{m}$, no vertex added after time $t_{1}$ has degree exceeding $t_{0}^{-2} t^{1 / 2}$ whp. 
Proof. Let $\mathcal{A}_{2}$ denote the event that some vertex added after time $t_{1}$ has degree exceeding $t_{0}^{-2} t^{1 / 2}$. Then we have

$$
\begin{aligned}
\operatorname{Pr}\left[\mathcal{A}_{2}\right] \leq \sum_{s=t_{1}}^{t} \operatorname{Pr}\left[d_{t}(s) \geq t_{0}^{-2} t^{1 / 2}\right] & =\sum_{s=t_{1}}^{t} \operatorname{Pr}\left[\left(d_{t}(s)\right)^{(3)} \geq\left(t_{0}^{-2} t^{1 / 2}\right)^{(3)}\right] \\
& \leq \sum_{s=t_{1}}^{t} t_{0}^{6} t^{-3 / 2} \mathrm{E}\left[d_{t}(s)^{(3)}\right]
\end{aligned}
$$

Using Lemma 1.3, this bound becomes

$$
\begin{aligned}
\operatorname{Pr}\left[\mathcal{A}_{2}\right] \leq \sum_{s=t_{1}}^{t} t_{0}^{6} t^{-3 / 2}(2 m)^{(3)} 2^{3 / 2}\left(\frac{t}{s}\right)^{3 / 2} & \leq(2 m)^{(3)} 2^{3 / 2} t_{0}^{6} \sum_{s=t_{1}}^{t} s^{-3 / 2} \\
& \leq(2 m)^{(3)} 2^{3 / 2} t_{0}^{6} t_{1}^{-1 / 2}=o(1) .
\end{aligned}
$$

Claim 2.3. In $G_{t}^{m}$, no vertex added before time $t_{1}$ has degree exceeding $t_{0}^{1 / 6} t^{1 / 2}$ whp.

Proof. Let $\mathcal{A}_{3}$ denote the event that some vertex added before $t_{1}$ has degree exceeding $t_{0}^{1 / 6} t^{1 / 2}$. We use Lemma 1.3 for a third moment argument as above:

$$
\begin{aligned}
\operatorname{Pr}\left[\mathcal{A}_{3}\right] & \leq \sum_{s=1}^{t_{1}}\left(t_{0}^{1 / 6} t^{1 / 2}\right)^{-3}(2 m)^{(3)} 2^{3 / 2}\left(\frac{t}{s}\right)^{3 / 2}=(2 m)^{(3)} 2^{3 / 2} t_{0}^{-1 / 2} \sum_{s=1}^{t_{1}} s^{-3 / 2} \\
& \leq(2 m)^{(3)} 2^{3 / 2} t_{0}^{-1 / 2}=o(1) .
\end{aligned}
$$

Claim 2.4. The $k$ highest degree vertices of $G_{t}^{m}$ are added before time $t_{1}$ and have degree $\Delta_{i}$ bounded by $t_{0}^{-1} t^{1 / 2} \leq \Delta_{i} \leq t_{0}^{1 / 6} t^{1 / 2}$ whp.

Proof.

(Upper bound on $\boldsymbol{\Delta}_{\boldsymbol{i}}$ ) ) By Claim 2.2, all vertices added after time $t_{1}$ have degree at most $t_{0}^{-2} t^{1 / 2}$ whp. Combining this with Claim 2.3, we have $\Delta_{1} \leq t_{0}^{1 / 6} t^{1 / 2}$ whp.

(Lower bound on $\boldsymbol{\Delta}_{i}$.) The conditions from Claims 2.1, 2.2, and 2.3 imply the lower bound. To see this, suppose the conditions of these claims are satisfied, but assume for contradiction that at most $k-1$ vertices added before $t_{1}$ have degree exceeding $t_{0}^{-1} t^{1 / 2}$. Then the total degree of vertices added before $t_{0}$ is less than $k\left(t_{0}^{1 / 6} t^{1 / 2}\right)+t_{0}\left(t_{0}^{-1} t^{1 / 2}\right) \leq 2 k t_{0}^{1 / 6} t^{1 / 2}$. But this contradicts the condition of Claim 2.1, which says the total degree of vertices added before $t_{0}$ is at least $t_{0}^{1 / 3} t^{1 / 2}$. 
(Added before $\boldsymbol{t}_{\mathbf{1}}$.) By Claim 2.2, all vertices added after time $t_{1}$ have degree at most $t_{0}^{-2} t^{1 / 2}$ whp. So the lower bound on $\Delta_{i}$ shows the $k$ highest degree vertices are added before time $t_{1}$ whp.

Claim 2.5. The $k$ highest degree vertices of $G_{t}^{m}$ have $\Delta_{i} \leq \Delta_{i-1}-t^{1 / 2} / f(t)$ whp.

Proof. Let $\mathcal{A}_{4}$ denote the event that there are two vertices among the first $t_{1}$ with degrees exceeding $t_{0}^{-1} t^{1 / 2}$ and within $t^{1 / 2} / f(t)$ of each other.

Let $p_{\ell, s_{1}, s_{2}}=\operatorname{Pr}\left[d_{t}\left(s_{1}\right)-d_{t}\left(s_{2}\right)=\ell \mid \overline{\mathcal{A}_{3}}\right]$, for $|\ell| \leq \sqrt{t} / f(t)$. Then

$$
\operatorname{Pr}\left[\mathcal{A}_{4} \mid \overline{\mathcal{A}_{3}}\right] \leq \sum_{1 \leq s_{1}<s_{2} \leq t_{1}} \sum_{\ell=-t^{1 / 2} / f(t)}^{t^{1 / 2} / f(t)} p_{\ell, s_{1}, s_{2}} .
$$

Since

$$
\begin{aligned}
p_{\ell, s_{1}, s_{2}} & \leq \sum_{r_{1}=t_{0}^{-1} t^{1 / 2}}^{t_{0}^{1 / 6} t^{1 / 2}} \sum_{d_{1}, d_{2}=1}^{2 t_{1}} p_{\left(s_{1}, s_{2}\right)}\left(\left(r_{1}, r_{1}-\ell\right) ;\left(d_{1}, d_{2}\right), t_{1}, t\right) \\
& \leq t_{0}^{1 / 6} t^{1 / 2} \sum_{d_{1}, d_{2}=1}^{2 t_{1}}\left(\begin{array}{c}
2 t_{0}^{1 / 6} t^{1 / 2} \\
d_{1}-1
\end{array}\right)\left(\begin{array}{c}
2 t_{0}^{1 / 6} t^{1 / 2} \\
d_{2}-1
\end{array}\right)\left(\frac{t_{1}+1}{t}\right)^{\left(d_{1}+d_{2}\right) / 2} e^{t_{0}+2+4 t_{0}^{1 / 6}} \\
& \leq t_{0}^{1 / 6} t^{1 / 2} \sum_{d_{1}, d_{2}=1}^{2 t_{1}}\left(2 t_{0}^{1 / 6} t^{1 / 2}\right)^{d_{1}+d_{2}-2}\left(t_{1}+1\right)^{2 t_{1}} t^{-\left(d_{1}+d_{2}\right) / 2} e^{2 t_{0}} \\
& \leq t_{0}^{1 / 6}\left(2 t_{1}\right)^{2} 2^{4 t_{1}} t_{0}^{2 t_{1} / 3}\left(t_{1}+1\right)^{2 t_{1}} e^{2 t_{0}} t^{-1 / 2} \\
& =o\left(t_{1}^{-2} t^{-1 / 2} f(t)\right)
\end{aligned}
$$

we have

$$
\operatorname{Pr}\left[\mathcal{A}_{4} \mid \overline{\mathcal{A}_{3}}\right] \leq \sum_{1 \leq s_{1}<s_{2} \leq t_{1}} \sum_{\ell=-t^{1 / 2} / f(t)}^{t^{1 / 2} / f(t)} p_{\ell, s_{1}, s_{2}}=o(1)
$$

So

$\operatorname{Pr}\left[\mathcal{A}_{4}\right]=\operatorname{Pr}\left[\mathcal{A}_{4} \mid \mathcal{A}_{3}\right] \operatorname{Pr}\left[\mathcal{A}_{3}\right]+\operatorname{Pr}\left[\mathcal{A}_{4} \mid \overline{\mathcal{A}_{3}}\right] \operatorname{Pr}\left[\overline{\mathcal{A}_{3}}\right] \leq \operatorname{Pr}\left[\mathcal{A}_{3}\right]+\operatorname{Pr}\left[\mathcal{A}_{4} \mid \overline{\mathcal{A}_{3}}\right]=o(1)$ 


\subsection{Proof of Theorem 1.2}

We partition the vertices into three sets; let $S_{i}$ be the vertices added after time $t_{i-1}$ and at or before time $t_{i}$, for

$$
t_{0}=0, \quad t_{1}=t^{1 / 8}, \quad t_{2}=t^{9 / 16}, \quad t_{3}=t
$$

To reduce the number of subscripts necessary, we use $G$ to denote the graph $G_{t}$.

For any graph $H$, we let $M_{H}$ denote the adjacency matrix of $H$, and we let $\lambda_{i}(H)$ denote the $i$ th largest eigenvalue of $M_{H}$. We will use the identity (Rayleigh's Principle)

$$
\lambda_{i}(H)=\min _{L} \max _{\mathbf{x} \in L, \mathbf{x} \neq 0} \frac{\mathbf{x}^{T} M_{H} \mathbf{x}}{\mathbf{x}^{T} \mathbf{x}}
$$

where $L$ ranges over all $(n-i+1)$-dimensional subspaces of $\mathbb{R}^{n}$. (See, for example, [Strang 88]).

Our approach, as in [Mihail and Papadimitriou 02], [Chung et al. 03a, Chung et al. 03b], is to show that whp $G$ contains a star forest $F$ with stars of degree asymptotic to the maximum degree vertices of $G$. Then we will show $G \backslash F$ has small eigenvalues. Then Rayleigh's Principle is sufficient to conclude that the large eigenvalues of $G$ cannot be too different from the large eigenvalues of $F$.

To do this, we need reasonable bounds on the degrees and codegrees in $G$. Recall that $d_{s}^{m}(r)$ is the degree at time $s$ of the vertex added at time $r$ with contractions of size $m$.

Claim 2.6. For any $\epsilon>0$ and any $f(t)$ with $f(t) \rightarrow \infty$ as $t \rightarrow \infty$ the following holds whp: for all $s$ with $f(t) \leq s \leq t$, for all vertices $v \in G_{s}^{m}$, if $v$ was added at time $r$, then $d_{s}^{m}(v) \leq s^{1 / 2+\epsilon} r^{-1 / 2}$.

Proof. We use Lemma 1.3 and the union bound. Let $\ell=\lceil 3 / \epsilon\rceil$.

$$
\begin{aligned}
\operatorname{Pr}\left[\bigcup_{s=f(t)}^{t}\right. & \left.\bigcup_{r=1}^{s}\left\{d_{s}^{m}(r) \geq s^{1 / 2+\epsilon} r^{-1 / 2}\right\}\right] \\
\leq & \sum_{s=f(t)}^{t} \sum_{r=1}^{s} \operatorname{Pr}\left[d_{s}^{m}(r) \geq s^{1 / 2+\epsilon} r^{-1 / 2}\right] \\
& =\sum_{s=f(t)}^{t} \sum_{r=1}^{s} \operatorname{Pr}\left[\left(d_{s}^{m}(r)\right)^{(\ell)} \geq\left(s^{1 / 2+\epsilon} r^{-1 / 2}\right)^{(\ell)}\right]
\end{aligned}
$$




$$
\begin{aligned}
& \leq \sum_{s=f(t)}^{t} \sum_{r=1}^{s} s^{-\ell(1 / 2+\epsilon)} r^{\ell / 2} \mathrm{E}\left[\left(d_{s}^{m}(r)\right)^{\ell}\right] \\
& \leq \sum_{s=f(t)}^{t} \sum_{r=1}^{s} s^{-\ell(1 / 2+\epsilon)} r^{\ell / 2}(2 m)^{(\ell)} 2^{\ell / 2}(s / r)^{\ell / 2} \\
& =(2 m)^{(\ell)} 2^{\ell / 2} \sum_{s=f(t)}^{t} s^{1-\epsilon \ell} .
\end{aligned}
$$

Since $\ell \geq 3 / \epsilon$

$$
\sum_{s=f(t)}^{t} s^{1-\epsilon \ell} \leq \int_{f(t)-1}^{\infty} x^{1-\epsilon \ell} d x=\frac{1}{\epsilon \ell-2}(f(t)-1)^{2-\epsilon \ell}=o(1) .
$$

Claim 2.7. Let $S_{3}^{\prime}$ be the set of vertices in $S_{3}$ that are adjacent to more than one vertex of $S_{1}$ in $G$. Then $\left|S_{3}^{\prime}\right| \leq t^{7 / 16}$ whp.

Proof. Let $\mathcal{B}_{1}$ be the event that the conditions of Claim 2.6 hold with $f(t)=t_{2}$ and $\epsilon=1 / 16$. Then for a vertex $v \in S_{3}$ added at time $s$,

$$
\operatorname{Pr}\left[\left|N(v) \cap S_{1}\right| \geq 2 \mid \mathcal{B}_{1}\right] \leq\left(\begin{array}{c}
m \\
2
\end{array}\right)\left(\frac{s^{1 / 2+\epsilon} t_{1}}{2 s-1}\right)^{2} \leq m^{2} s^{-7 / 8} t^{1 / 4} .
$$

Let $X$ denote the number of $v \in S_{3}$ adjacent to more than one vertex of $S_{1}$. Then

$$
\mathrm{E}\left[X \mid \mathcal{B}_{1}\right] \leq \sum_{s=t_{2}+1}^{t} m^{2} s^{-7 / 8} t^{1 / 4} \leq m^{2} t^{1 / 4} \int_{t_{2}}^{t} x^{-7 / 8} d x \leq 8 m^{2} t^{3 / 8}
$$

We finish the claim with Markov's inequality,

$$
\operatorname{Pr}\left[X \geq t^{7 / 16} \mid \mathcal{B}_{1}\right] \leq E\left[X \mid \mathcal{B}_{1}\right] / t^{7 / 16}=o(1)
$$

Now, let $F \subseteq G$ be the star forest consisting of edges between $S_{1}$ and $S_{3} \backslash S_{3}^{\prime}$.

Claim 2.8. Let $\Delta_{1} \geq \Delta_{2} \geq \cdots \geq \Delta_{k}$ denote the degrees of the $k$ highest degree vertices of $G$. Then $\lambda_{i}(F)=(1-o(1)) \Delta_{i}^{1 / 2}$ whp. 
Proof. Let $H$ be the star forest $H=K_{1, d_{1}} \cup K_{1, d_{2}} \cup \cdots \cup K_{1, d_{k}}$, with $d_{1} \geq d_{2} \geq$ $\cdots \geq d_{k}$. Then for $i=1, \ldots, k, \lambda_{i}(H)=d_{i}^{1 / 2}$. So it is sufficient to show that $\Delta_{i}(F)=(1-o(1)) \Delta_{i}(G)$ for $i=1, \ldots, k$.

Claim 2.4 shows that the $k$ highest degree vertices of $G$ are added before time $t_{1}$, so these vertices are all in $F$. The only edges to these vertices that are not in $F$ are those added before time $t_{2}$ and those incident to $S_{3}^{\prime}$. By Theorem 1.1, we have $\Delta_{1}\left(G_{t_{2}}^{m}\right) \leq t_{2}^{7 / 9}=t^{7 / 16}$ and, also by Theorem 1.1, $\Delta_{i}(G) \geq t^{1 / 2} / \log t$ for $i=1, \ldots, k$, whp. Claim 2.7 says that whp $\left|S_{3}^{\prime}\right| \leq t^{7 / 16}$, and so whp

$$
\Delta_{i}(F) \geq \Delta_{i}(G)-t^{7 / 16}-m t^{7 / 16}=(1-o(1)) \Delta_{i}(G) .
$$

Let $H=G \backslash F$. We now show that $\lambda_{1}(H)$ is $o\left(\lambda_{k}(F)\right)$. This completes the proof of Theorem 1.2 because, for any subspace $L$, we have

$$
\max _{\mathbf{x} \in L, \mathbf{x} \neq 0} \frac{\mathbf{x}^{T} M_{G} \mathbf{x}}{\mathbf{x}^{T} \mathbf{x}}=\max _{\mathbf{x} \in L, \mathbf{x} \neq 0} \frac{\mathbf{x}^{T} M_{F} \mathbf{x}}{\mathbf{x}^{T} \mathbf{x}} \pm O\left(\max _{\mathbf{x} \neq 0} \frac{\mathbf{x}^{T} M_{H} \mathbf{x}}{\mathbf{x}^{T} \mathbf{x}}\right),
$$

and so, for $i \leq k$, Rayleigh's Principle gives $\lambda_{i}(G)=\lambda_{i}(F)(1 \pm o(1))$.

Claim 2.9. $\lambda_{1}(H) \leq 6 m t^{15 / 64} w h p$.

Proof. We bound the eigenvalues of $H$ in six parts. Let

$$
H_{i}=H\left[S_{i}\right], \quad H_{i j}=H\left(S_{i}, S_{j}\right),
$$

where $H[S]$ is the subgraph of $H$ induced by the vertex set $S$, and $H(S, T)$ is the subgraph containing only edges with one vertex in $S$ and the other in $T$.

To bound $\lambda_{1}\left(H_{i}\right)$, we use the fact that the maximum eigenvalue of a graph is at most the maximum degree of the graph. This is easily verified from (2.1).

We use Claim 2.6 with $f(t)=t_{1}$ and $\epsilon=1 / 64$ to conclude that whp

$$
\begin{array}{lllll}
\lambda_{1}\left(H_{1}\right) & \leq \Delta_{1}\left(H_{1}\right) & =\max _{v \leq t_{1}}\left\{d_{t_{1}}^{m}(v)\right\} & \leq t_{1}^{1 / 2+\epsilon} & =t^{33 / 512}, \\
\lambda_{1}\left(H_{2}\right) & \leq \Delta_{1}\left(H_{2}\right) & \leq \max _{t_{1} \leq v \leq t_{2}}\left\{d_{t_{2}}^{m}(v)\right\} & \leq t_{2}^{1 / 2+\epsilon} t_{1}^{-1 / 2}=t^{233 / 1024} \\
\lambda_{1}\left(H_{3}\right) & \leq \Delta_{1}\left(H_{3}\right) & \leq \max _{t_{2} \leq v \leq t_{3}}\left\{d_{t_{3}}^{m}(v)\right\} & \leq t_{3}^{1 / 2+\epsilon} t_{2}^{-1 / 2}=t^{15 / 64} .
\end{array}
$$

To bound $\lambda_{1}\left(H_{i j}\right)$, we begin by considering the case $m=1$. Then, for $i<j$, each vertex in $S_{j}$ has at most one edge in $H_{i j}$, so $H_{i j}$ is a star forest. As observed in Claim 2.8, the eigenvalues of a star forest are directly related to the degrees of the stars.

When $m>1$, we let $G^{\prime}$ denote a preferential attachment graph with $t$ edges and $m=1$. Recall that by contracting vertices $\{(i-1) m+1, \ldots, i m\}$ into a 
single vertex $i$, we obtain a graph identically distributed with $G$. There is a simple representation of this observation in terms of linear algebra: we can write the adjacency matrix of $G$ in terms of the adjacency matrix of the graph $G^{\prime}$ :

$$
M_{G}=C_{m}^{T} M_{G^{\prime}} C_{m}
$$

where $C_{m}$ is the $t \times t / m$ matrix with $i$ th column

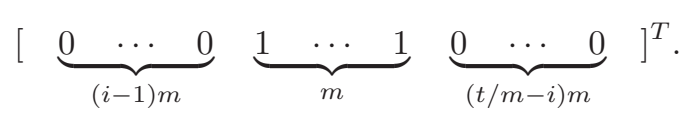

Similarly, we can write the adjacency matrix of $H_{i j}$ in terms of the adjacency matrix of $H_{i j}^{\prime}$ using this "contraction matrix" $C_{m}$.

Note that for $\mathbf{y}=C_{m} \mathbf{x}$, we have $\mathbf{y}^{T} \mathbf{y}=m\left(\mathbf{x}^{T} \mathbf{x}\right)$. So

$$
\begin{aligned}
\lambda_{1}\left(H_{i j}\right) & =\max _{\mathbf{x} \neq 0} \frac{\mathbf{x}^{T} M_{H_{i j}} \mathbf{x}}{\mathbf{x}^{T} \mathbf{x}}=\max _{\mathbf{x} \neq 0} \frac{\mathbf{x}^{T} C_{m}^{T} M_{H_{i j}^{\prime}} C_{m} \mathbf{x}}{\mathbf{x}^{T} \mathbf{x}}=\max _{\mathbf{y}: \mathbf{y}=C_{m} \mathbf{x} \neq 0} m \frac{\mathbf{y}^{T} M_{H_{i j}^{\prime}} \mathbf{y}}{\mathbf{y}^{T} \mathbf{y}} \\
& \leq m \max _{\mathbf{y} \neq 0} \frac{\mathbf{y}^{T} M_{H_{i j}^{\prime}} \mathbf{y}}{\mathbf{y}^{T} \mathbf{y}}=m \lambda_{1}\left(H_{i j}^{\prime}\right) .
\end{aligned}
$$

We use Claim 2.6 with $f(t)=t_{1}$ and $\epsilon=1 / 64$ as above to conclude that whp

$$
\begin{array}{llll}
\Delta_{1}\left(H_{12}^{\prime}\right) & =\max _{v \leq t_{2}}\left\{d_{t_{2}}^{1}(v)\right\} & \leq t_{2}^{1 / 2+\epsilon} & =t^{297 / 1024} \\
\Delta_{1}\left(H_{23}^{\prime}\right) & =\max _{t_{1} \leq v \leq t_{3}}\left\{d_{t_{3}}^{1}(v)\right\} & \leq t_{3}^{1 / 2+\epsilon} t_{1}^{-1 / 2} & =t^{29 / 64} .
\end{array}
$$

Finally, all edges in $H_{13}^{\prime}$ are between $S_{1}$ and $S_{3}^{\prime}$, so Claim 2.7 shows that $\Delta_{1}\left(H_{13}^{\prime}\right) \leq t^{7 / 16}$ whp.

We now conclude that whp

$$
\lambda_{1}\left(H_{i j}\right) \leq m \lambda_{1}\left(H_{i j}^{\prime}\right) \leq m \Delta_{1}\left(H_{i j}^{\prime}\right)^{1 / 2} \leq m t^{15 / 64},
$$

and so whp

$$
\lambda_{1}(H) \leq \sum_{i=1}^{3} \lambda_{1}\left(H_{i}\right)+\sum_{i<j} \lambda_{1}\left(H_{i j}\right) \leq 6 m t^{15 / 64}
$$

Acknowledgments. We would like to thank the referee whose suggestion concerning Lemma 1.3 has considerably simplified part of the paper. The first author was supported in part by NSF VIGRE Grant DMS-9819950. The second author was supported in part by NSF Grant CCR-0200945. 


\section{References}

[Albert et al. 99] R. Albert, A. Barabási, and H. Jeong. "Diameter of the World Wide Web." Nature 401 (1999), 103-131.

[Aiello et al. 00] W. Aiello, F. R. K. Chung, and L. Lu. "A Random Graph Model for Massive Graphs." In Proc. of the 32nd Annual ACM Symposium on the Theory of Computing, pp. 171-180. New York: ACM Press, 2000.

[Barabási and Albert 99] A. Barabási and R. Albert. "Emergence of Scaling in Random Networks." Science 286 (1999), 509-512.

[Broder et al. 00] A. Broder, R. Kumar, F. Maghoul, P. Raghavan, S. Rajagopalan, R. Stata, A. Tomkins, and J. Wiener. "Graph Structure in the Web." In Proc. of the 9th Intl. World Wide Web Conference, pp. 309-320. Amsterdam: NorthHolland Publishing Company, 2000.

[Buckley and Osthus] G. Buckley and D. Osthus. "Popularity Based Random Graph Models Leading to a Scale-Free Degree Distribution." To appear in Discrete Mathematics.

[Bollobás and Riordan] B. Bollobás and O. Riordan. "The Diameter of a Scale-Free Random Graph." To appear in Combinatorica.

[Bollobás and Riordan 02] B. Bollobás and O. Riordan. "Mathematical Results on Scale-Free Random Graphs." In Handbook of Graphs and Networks, pp. 1-34. Berlin: Wiley-VCH, 2002.

[Bollobás et al. 01] B. Bollobás, O. Riordan, J. Spencer, and G. Tusanády. "The Degree Sequence of a Scale-Free Random Graph Process." Random Structures and Algorithms 18 (2001), 279-290.

[Cooper and Frieze 01] C. Cooper and A. M. Frieze. "A General Model of Undirected Web Graphs.” In Proc. of ESA, pp. 500-511. London: Springer-Verlag, 2001.

[Chung et al. 03a] F. R. K. Chung, L. Lu, and V. Vu. "Eigenvalues of Random Power Law Graphs." Annals of Combinatorics 7 (2003), 21-33.

[Chung et al. 03b] F. R. K. Chung, L. Lu, and V. Vu. "The Spectra of Random Graphs with Expected Degrees." Proceedings of National Academy of Sciences 100:11 (2003), 6313-6318.

[Drinea et al. 01] E. Drinea, M. Enachescu, and M. Mitzenmacher. "Variations on Random Graph Models for the Web." Technical report, Harvard University, 2001.

[Erdös and Rényi 59] P. Erdös and A. Rényi. "On Random Graphs I." Publicationes Mathematicae Debrecen 6 (1959), 290-297.

[Faloutsos et al. 99] M. Faloutsos, P. Faloutsos, and C. Faloutsos. "On Power-Law Relationships of the Internet Topology." In SIGCOMM, pp. 251-262. New York: ACM Press, 1999.

[Hayes 00] B. Hayes. "Graph Theory in Practice: Part II." American Scientist 88 (2000), 104-109. 
[Kleinberg et al. 99] J. M. Kleinberg, R. Kumar, P. Raghavan, S. Rajagopalan, and A. S. Tomkins. "The Web as a Graph: Measurements, Models and Methods." In Proc. 19th ACM SIGACT-SIGMOD-AIGART Symp. Principles of Database Systems, PODS, pp. 1-10. New York: ACM Press, 2000.

[Kumar et al. 99] R. Kumar, P. Raghavan, S. Rajagopalan, and A. Tomkins. "Trawling the Web for Emerging Cyber-Communities." Computer Networks (Amsterdam, Netherlands: 1999), 31:11-16 (1999), 1481-1493.

[Kumar et al. 00] R. Kumar, P. Raghavan, S. Rajagopalan, D. Sivakumar, A. Tomkins, and E. Upfal. "Stochastic Models for the Web Graph." In FOCS: IEEE Symposium on Foundations of Computer Science (FOCS), pp. 57-65. Washington, DC: IEEE Computer Society, 2000.

[Mitzenmacher 04] M. Mitzenmacher. "A Brief History of Generative Models for Power Law and Lognormal Distributions." Internet Mathematics 1:2 (2004), 226-251.

[Mihail and Papadimitriou 02] M. Mihail and C. H. Papadimitriou. "On the Eigenvalue Power Law." In Randomization and Approximation Techniques, 6th International Workshop, pp. 254-262. London: Springer-Verlag, 2002.

[Simon 55] H. A. Simon. "On a Class of Skew Distribution Functions." Biometrika 42:3/4 (1955), 425-440.

[Strang 88] G. Strang. Linear Algebra and Its Applications. San Diego, CA: Harcourt Brace Jovanovich Publishing, 1988.

[Watts 99] D. J. Watts. Small Worlds: The Dynamics of Networks Between Order and Randomness. Princeton, NJ: Princeton University Press, 1999.

[Yule 25] G. Yule. A Mathematical Theory of Evolution Based on the Conclusions of Dr. J.C. Willis. Philosophical Transactions of the Royal Society of London (Series B) 213 (1925), 21-87.

Abraham Flaxman, Department of Mathematical Sciences, Carnegie Mellon University, Pittsburgh, PA, 15213 (abie@cmu.edu)

Alan Frieze, Department of Mathematical Sciences, Carnegie Mellon University, Pittsburgh, PA, 15213 (alan@random.math.cmu.edu)

Trevor Fenner, School of Computer Science, Birkbeck College, University of London, Malet Street, London WC1E 7HX, UK (trevor@dcs.bbk.ac.uk)

Received June 19, 2003; accepted October 2, 2003. 